\title{
Fridolin Schuler (1832-1903), ein Pionier der Arbeitshygiene im 19. Jahrhundert
}

\author{
Von Erich Auer und Heinrich Buess *, Basel
}

\section{Biographisches}

Am 1. April 1832 wurde Fridolin Schuler im Pfarrhaus des glarnerischen Dorfes Bilten geboren. Er verbrachte eine frohe Jugendzeit und war ein aufgeweckter Schüler, der trotz strenger Lehrmeister immer wieder Zeit fand, seinen Vater bei dessen Tätigkeit als Pfarrherr zu begleiten. Dadurch lernte der junge Schuler schon recht früh die Gebräuche und Lebensweise der Dorfbevölkerung kennen und wurde mit hunderterlei Anliegen der Leute bekannt. 1848 besuchte er die Kantonsschule in Aarau und zog 1851 als Medizinstudent an die Universität Zürich. Einen Teil seiner Studien verbrachte er in Würzburg und besuchte auf Reisen München, Prag, Stuttgart und Dresden. In seine Heimat zurückgekehrt, bestand er das kantonale Examen als Arzt. Kurz hernach verehelichte er sich und begann in Mollis seine Praxistätigkeit. Es sollte eine schwere, aufopfernde Zeit werden.

Einerseits hatte er sich mit der Quacksalberei, die damals von der Landsgemeinde gebilligt war, auseinanderzusetzen, und andererseits erkannten seine älteren Kollegen recht bald, daß hier ein befähigter, mit großem Wissen ausgerüsteter Arzt tätig war, welcher in seinen Untersuchungs- und Therapiemethoden ganz neue Wege einschlug. Sie hielten demzufolge mit der Opposition nicht zurück. Gar bald erfreute sich aber Schuler bei seinen Patienten größter Beliebtheit, und so war es nicht erstaunlich, daß sich seine Tätigkeit als Arzt weit über Mollis hinaus bis in die entlegensten Bergtäler und Weiler erstreckte. Trotz dieser enorme Kräfte verzehrenden Praxistätigkeit befaßte sich Schuler mit Vorliebe mit hygienischen und gewerbemedizinischen Studien. Eifrig beschäftigte er sich auch mit der Ernährungslehre, sah er doch bei seinen Krankenvisiten, wie kümmerlich die Bevölkerung lebte.

Im öffentlichen Leben bekleidete der angesehene Glarner Arzt unzählige Stellen, die er mit der ihm eigenen Gewissenhaftigkeit und größtem Ver-

\footnotetext{
* Fabrikärztlicher Dienst der Ciba AG, Basel.
} 
antwortungsbewußtsein ausfüllte. Als Mediziner war er Armenarzt, Mitglied der Sanitätskommission des Kantons, Gerichtsarzt, Mitglied der Konkordatsprüfungskommission in Basel, d.h. der Vorläuferin der eidgenössischen Prüfungsbehörde. Aber als Kantonsschulrat, als Landrat und im Appellationsgericht schätzte man das klare, kurze Urteil Schulers. Da ihn die hygienische Seite des ärztlichen Wirkens ganz besonders anzog, war es auch nicht verwunderlich, daß er das Amt eines glarnerischen Fabrikinspektors versah und an mehreren Hygienekongressen im Auslande als Referent anzutreffen war.

Verständlicherweise führte dieses außerordentlich große Arbeitspensum - neben der Erledigung der täglichen Praxis - zu einer vorzeitigen Ermüdung. Schuler erkannte, daß seine Kräfte für die schwere Arbeit nicht mehr ausreichten und war glücklich, im Jahre 1877 diese einem Neffen übergeben zu können, der vorher bei ihm als Assistent tätig gewesen war. Von dieser schweren Last befreit, hoffte er nun, sich ganz seinen privaten Studien widmen zu können. Doch es sollte anders kommen!

Ein Jahr später, 1878, wurde er an die Stelle eines eidgenössischen Fabrikinspektors gewählt, und damit gewann sein Leben eine ganz neue Wendung. Es bedeutet dies das Ende seiner spezifisch ärztlichen Tätigkeit.

Sein Wirken als Fabrikinspektor war von so umwälzender Bedeutung, daß wir später genauer darauf zu sprechen kommen werden.

Am letzten Tage des Jahres 1901 reichte Schuler sein Entlassungsgesuch aus der eidgenössischen Fabrikinspektion ein. Mit seinem siebzigsten Geburtstage durfte er den Dank von allen Seiten entgegennehmen. Der einfachste Arbeiter, die Fabrikanten, Kanton und Bundesrat wußten um die vortrefflichen Dienste, die der Jubilar als Mensch und Arzt geleistet hatte. Die staatswirtschaftliche Fakultät der Universität Zürich verlieh ihm 1902 den Titel des Ehrendoktors. Schon 17 Jahre früher hatte ihn die Medizinische Fakultät Basel ehrenhalber promoviert. Damit war das Maß der aufopfernden Tätigkeit für das Wohl der arbeitenden Bevölkerung erschöpft. Die Tage des stillen Greisenalters waren sehr spärlich. Schuler starb schon im Jahre 1903. Ein vornehmer, gütiger und - für die damalige Zeit besonders bemerkenswert - sozial denkender Mensch, aber auch ein hervorragender Arzt und Hygieniker hinterließ ein Erbe, aus welchem wir bis in unsere Tage hinein reichlich Nutzen ziehen konnten. 


\section{Fabrikinspektion}

Wir wissen, daß neben der Ausübung der ärztlichen Praxis die Studien über Hygiene zu den Lieblingsbeschäftigungen Schulers gehörten. Als Arzt und Fabrikinspektor hatte er sich zur Aufgabe gemacht, die Bedingungen, unter denen seine Pflegebefohlenen lebten und arbeiteten, möglichst genau kennenzulernen und sich mit allen Einwirkungen vertraut zu machen, welche die Industrie auf die ganze Existenz unserer Bevölkerung hat. Die Zustände, die Schuler antraf, waren so erbärmlich und menschenunwürdig, daß man sich heute kaum ein Bild davon machen kann. Um die nachfolgenden Werke Schulers zu verstehen, ist die Kenntnis der Ausgangslage der Fabrikinspektorentätigkeit unentbehrlich. Hören wir aus einem der ersten Berichte (1872), die er über den Besuch in den glarnerischen Baumwollfabriken verfaßte:

«Die Menschen arbeiten in unreinlichen, auf das Primitivste eingerichteten Räumen, schlecht gelüftet, in Staub und Dampf, bei mangelhafter Beleuchtung, Tag und Nacht, Werktags und Sonntags. Die Entlöhnung reicht nicht zur zweckmäßigen Ernährung aus, Kinder und Frauen sind dem Betrieb einverleibt und verrichten Männerarbeit, niemand kümmert sich um die Schwangeren, die kurz nach ihrer Niederkunft wieder zur Arbeit zurückzukehren haben, in den Spinnereien sitzen halbnackte schweißtriefende Arbeiter und Scharen jämmerlich aussehender Kinder. An den Schutz des Menschen und an die Möglichkeit von Gesundheitsschädigungen wird nicht gedacht.»

Über die Färbereilokale schreibt Schuler: «... es sind bald enge, finstere Löcher, wo weder Luft noch Licht recht eindringen kann, wo Dampf und Nebel den Luftraum bei einigermaßen kühler Witterung erfüllt, wo der Fußboden aus holperigem Pflaster oder zerbrochenen Steinplatten besteht, in deren Zwischenräumen alle möglichen hergeschwemmten Substanzen sich ablagern, wo von eigentlichen Ventilationseinrichtungen keine Rede ist, dagegen die Luft aus dem Freien bei rauhem Wetter lebhaft durchzieht, ja vielleicht der durch das Arbeitslokal durchfließende Bach stets einen kalten Luftzug bedingt und im Winter die Temperatur auf einem außerordentlich niedrigen Grade hält ...»

Welche gesundheitsschädigenden Auswirkungen diese Verhältnisse in sich bargen, hatte Schuler sofort erkannt. Er intensivierte seine Bemühungen zum Schutze von Leben und Gesundheit der Arbeiter. Auf Grund seiner hygienischen Kenntnisse machte er erste Vorschläge zum Um- oder Neubau 
der Fabriken, verfaßte Veröffentlichungen, in denen er die grundlegenden Begriffe über Licht, Wärme und Luft in den Arbeitsräumen allgemein definierte und verständlichmachte und stellte Forderungen auf, die erfüllt werden müßten, um die Gesundheitsschädigungen der Arbeiter auszuschließen.

Dem Mißbrauch der Kinder als Arbeitskräfte wirkte er durch einen Gesetzesvorschlag entgegen, welcher schulpflichtige Kinder von der Arbeit in den Spinnereien ausschloß und für ältere den Schichtenwechsel vorsah.

Es fehlte nicht an vernichtender Kritik und beträchtlicher Opposition. Die glarnerischen Fabrikanten beschwerten sich wegen der Konkurrenzunfähigkeit, des Produktionsausfalles infolge der verkürzten Arbeitszeiten und wiesen auf das Sinken der Arbeitslöhne hin. Schuler bezeichnete seine damalige Lage mit dem Ausdruck «Feinde ringsum!» Durch seinen persönlichen Einsatz wußte er aber den Verhältnissen Rechnung zu tragen und dennoch konsequent den Geltungsbereich des Gesetzes durchzusetzen.

Dieses Gesetz allein hätte nicht ausgereicht, die schweren Schädigungen der Arbeiterbevölkerung zu bekämpfen. Mit Fridolin Schuler war aber ein Mann zum Fabrikinspektor bestimmt worden, der durch sein fein ausgebildetes, soziales Denken, seinen Sinn für die Gefahren des arbeitenden Menschen und nicht zuletzt dank seiner medizinischen Kenntnisse und Erfahrungen es verstanden hat, die wirklichen Ursachen der großen Not zu erkennen.

In beispiellosem Einsatz besuchte der Glarner Arzt in 127 Tagen 286 Etablissemente, die 105 verschiedenen Industriezweigen angehörten. Daraus resultierte ein eindrückliches Bild der damaligen Verhältnisse in den Fabriken, die er sorgfältig studierte, und manche Verbesserungen in der Handhabung des Fabrikgesetzes waren die Früchte dieser Besichtigungen. Damit ermöglichte Schuler wertvolle Ergänzungen und Erweiterungen der allgemeinen Arbeiterschutzgesetzgebung in der Schweiz.

\section{Technische Unfallverhütung}

War nun in bezug auf den Einsatz der Arbeitskräfte, auf die Arbeitsdauer und Arbeitsräume etwas Disziplin und Ordnung geschaffen worden, so galt es jetzt, den Arbeiter am Arbeitsplatz zu schützen und zu erziehen. Trotz Mangel an technischem Wissen scheute Schuler vor dem Problem der technischen Unfallverhütung nicht zurück. Da es überall an den notwendigen Schutzmitteln fehlte, mußten alle zuerst erfunden, konstruiert und 
ausprobiert werden. Hatte er in mühsamer Kleinarbeit einen Schutz erfunden und bereit, so erforderte das psychologische Geschick des Glarners, gegen die Abneigung der Fabrikbesitzer anzukämpfen, welche solche kostspieligen Einrichtungen an den Maschinen und Apparaten nicht anbringen lassen wollten, da dadurch ohnehin die Produktionsleistung verringert würde.

Unzählige Besuche bei den Fabrikherren waren notwendig, um diese über ihren Irrtum aufzuklären. Hier hatte Schuler harte Pionierarbeit zu leisten. Er widmete aber diesem Gebiet des Arbeiterschutzes sein ganz besonderes Interesse, weil er schon recht früh erkannte, daß es mit der technischen Unfallverhütung allein nicht sein Bewenden haben konnte. Die Aufklärung der Fabrikbesitzer über die psychologische Unfallverhütung stellte gleichzeitig eine Notwendigkeit dar. Mittels Rundschreiben, Abhandlungen und anderen Schriften hoffte auch er diesen Zweig des Arbeiterschutzes bekanntzumachen. Das Echo seiner Bemühungen war zu jener Zeit bei Fabrikanten wie auch bei den Arbeitern ein recht schwaches. Schulers Erkenntnis und Weitsicht erlangten erst viel später die besondere Beachtung.

An der Landesausstellung von 1883 in Zürich warb der Glarner Fabrikinspektor mit Erfolg für seine Sache, indem er eine Spezialausstellung über Material und Methoden der Unfallverhütung organisierte. Der Bericht über das Gezeigte lautete recht zuversichtlich. Es wurde ausgeführt: «Soviel ist gewiß, daß die kleine Ausstellung den Beweis erbracht hat, daß in der Schweiz zum Mindesten verhältnismäßig ebenso viel für den Arbeiterschutz geleistet worden ist wie in irgend einem anderen industriellen Lande und dies ist umso höher anzuschlagen, als bisher keinerlei Institut zur Förderung dieser Art von Fortschritt bestand ...» Mit dem Material jener Ausstellung bildete Schuler den Anfang der Gewerbehygienischen Sammlung an der «Eidgenössischen Technischen Hochschule» in Zürich. In Zusammenarbeit mit dem großen Arzte Laurenz Sonderegger (1820-1895) setzte sich Schuler für die Einführung eines gewerbehygienischen Unterrichtes an der eidgenössischen Hochschule ein. Dieser Plan fand seine Verwirklichung im Jahre 1890.

\section{Erforschung und Bekämpfung der Berufskrankheiten}

Von größtem Wert und besonders glücklich war das Wirken Schulers in der Erforschung der Berufskrankheiten und ihrer Bedeutung bei der Entwicklung der modernen Arbeitshygiene. In umfassenden Studien wies er die 
schädigenden Einflüsse der Arbeitsprozesse in den Fabriken nach. Hatte er schon als sozial denkender Mensch mit der Einführung der Arbeiterschutzgesetzgebung gegen die lange Arbeitszeit Erfolg und als Hygieniker wesentlichen Anteil an der Verbesserung der allgemeinen Arbeitsbedingungen in den Fabriken, so kämpfte er nun als Arzt gegen die durch die Arbeitsprozesse bedingten Schädigungen. Bereits in der zweiten Hälfte der fünfziger Jahre des vergangenen Jahrhunderts war ihm bewußt geworden, daß die Wirkung der meist arsenhaltigen Chemikalien und Farben in den Kattundruckereien verheerende Folgen auf die Gesundheit der Arbeiter hatten. Schulers große Verdienste auf dem Gebiete der Gewerbemedizin rechtfertigen die Wiedergabe einiger Zeilen aus der Veröffentlichung über die glarnerische Baumwollindustrie, wo er im Kapitel über «Färben und Drucken und seine Schädlichkeiten» u.a. ausführt: «Das Terpentinöl wird als Lösungsmittel verwendet beim Druck einer gewissen meergrünen Farbe, dem sogenannten Giftgrün, dessen färbenden Bestandteil das ölsaure Kupferoxyd ausmacht. Ich hatte oft Gelegenheit, die schädliche Wirkung der Terpentinöldämpfe zu beobachten. Die betreffenden Arbeiter magerten auffallend $a b$, wurden blaß, verloren allen Appetit, klagten über beständigen Durst ohne Bedürfnis nach vieler Flüssigkeit, der Stuhl wurde unregelmäßig, meist retardiert, der Urin dunkler gefärbt. Der Puls wurde schneller, die Kranken klagten über die Schlaffheit aller Glieder, Kraftlosigkeit, schnelles Außeratemkommen, dumpfen Kopf, selbst halbbetäubten Zustand. Gewöhnlich litten sie an Augenbrennen in Folge Conjunctivitis. Die Genesung erfolgte erst, wenn sie einige Zeit ihre Arbeit ausgesetzt hatten ...

Schuler setzte seine Forschertätigkeit auf diesem Gebiete fort und klärte die Wirkungen von Chlor, Blei, Quecksilber und vor allem die Anilinintoxikationen ab. Neben den stofflichen Schädigungen untersuchte er die verschiedenen Arbeitsphasen und -prozesse, welche die Schädigungen auslösten. Abschließend stellte er fest, wie auf verschiedenartige Weise die Affektionen der Arbeiter durch diese Giftstoffe bedingt sein können. Mit unglaublicher Weitsicht erkannte und forderte er schon damals, daß die Ärzteschaft von ihren eigenen Erfahrungen aus dazu beitragen könnte, seine lückenhaften Beobachtungen zu ergänzen. Mit diesen Untersuchungen wurde Schuler zum eigentlichen Lehrer für die Bekämpfung der Berufskrankheiten. Innerhalb der mehrköpfigen eidgenössischen Fabrikinspektion hatte er in medizinischen Belangen eine absolute Führerrolle inne. So gingen zwei der wesentlichsten und weitreichendsten Verordnungen, nämlich diejenige über das Verbot des gelben Phosphors in der Zündholzindustrie 
und über das Verbot der Bleigewichte an den Jaccard-Webstühlen, auf seine Initiative und Warnung zurück. Auf diese Weise hatte der Glarner Arzt gute und umfassende Anfänge einer Arbeitsmedizin in der Schweiz gelegt. Leider fanden diese Erkenntnisse auf medizinischem Gebiete während vieler Jahre keine fachmännische Unterstützung mehr. Volle 28 Jahre wurde auch von staatlicher Seite nichts unternommen, was zur eigentlichen aktiven Bekämpfung der Berufskrankheiten hätte dienen können. Das Verständnis für eine solche Verhütungsaufgabe war bei den Arbeitgebern nicht vorhanden und stieß in der Durchführung oftmals auf Schwierigkeiten wirtschaftlicher und persönlicher Art. Erst im Jahre 1930 erkannte der Staat die Bedeutung der Medizin zur Hebung und Förderung der Arbeitshygiene und erließ die entsprechenden Weisungen.

\section{Lebensweise der Fabrikarbeiter}

Trotz aller Rückständigkeit und Unaufgeschlossenheit von seiten der Fabrikherren und zum Teil des Staates baute Schuler seine Vorstellung über die Ausmaße der modernen Arbeitsmedizin unentwegt weiter aus. Seine Erkenntnis scheint deshalb vollkommen verständlich, daß die Lösung der technischen, hygienischen und gesetzgeberischen Probleme einerseits und die Aufdeckung der Schädigungsquellen andererseits nicht für einen genügenden Arbeiterschutz Gewähr bieten. Er stellte fest, daß die Bestrebungen, die Gesundheitsverhältnisse der Fabrikbevölkerung zu verbessern, nach zwei Seiten hin gerichtet sein müßten. So verlangte er den genügenden Schutz des Arbeiters innerhalb der Fabrik und die Ermöglichung eines gesundheitsgemäßen Lebens außerhalb der Arbeitszeit. In ganz besonderem Maße arbeitete er an den Wandlungen des Wertbegriffes "Arbeiter» und befaßte sich mit der zunehmenden Bedeutung des Faktors Mensch. Es brauchte eine gute Dosis Mut und Sicherheit in einer Zeit, da von der Arbeitgeberschaft der Arbeiter nichts als «billige Kraft» war, sich in die Höhle des Löwen zu begeben und in unzähligen Voten und Besprechungen die Fabrikherren von der dringenden Notwendigkeit einer freiwilligen Verbesserung der Arbeiterverhältnisse außerhalb der Fabrik zu überzeugen und sie zur Mithilfe zu gewinnen.

Damit schuf der Glarner Arzt die Basis für die Entwicklung der Sozialmedizin in der Schweiz. Wie dringend notwendig dies war, ist aus Schulers Schilderung über die damaligen Wohnverhältnisse in seinem Heimatkanton Glarus erfaßbar. Er sagt darüber aus: «Die beschränkten Wohnräume 
der Fabrikarbeiter bedingen den Gebrauch von zweischläfrigen Betten. Die Überfüllung der Wohnungen nimmt jedes Jahr zu. Während im Kanton Zürich 86, in Bern gar nur 66 Seelen auf 100 bewohnte Räumlichkeiten kommen, in der ganzen Schweiz durchschnittlich 81, trifft es bei uns 97! Enge, niedrige Schlafzimmer, die 6 bis 8 Menschen beherbergen, sind nicht selten. Dasselbe ist mit den Stuben der Fall, die Abends und Feiertags für ein Dutzend und mehr Personen rauchender Männer, bettnässender Kinder Raum gewähren. Noch schlimmer ist, daß in den letzten Jahren so viele Parterre und oberflächlich gelegene Kellerräume, die früher als Gemüsekeller oder Vorratskeller dienten, oder auch allerlei luftige, undichte Anbauten als Wohnungen eingerichtet wurden. Wie oft hängt zollanger Schimmel herunter von den Wänden, wenn ein Bett von seiner Seite gerückt wird. Wie oft verpestet wahrer Modergeruch den Wohnraum.»

Schon während seiner Praxisjahre beschäftigte Schuler die Ernährungslehre sehr stark, denn auf seinen Krankenvisiten sah er nur zu oft, wie erbärmlich karg und wie wenig rationell der Kochtopf gefüllt war. So hielt er es für eine soziale Pflicht, die Frauen zum richtigen und günstigen Einkauf der Nahrungsmittel zu erziehen und auf Grund seines Wissens eine sachgemäße Zubereitung zu empfehlen. Unter seinen zahlreichen sozialen Einrichtungen seien lediglich die Eröffnung von Konsumvereinen, Suppenanstalten und Arbeiterküchen erwähnt. Noch mannigfache Möglichkeiten zur menschlicheren Gestaltung des Arbeiterlebens, zum Ausbau der geistigen Hygiene erkannte Schuler, die er neben seinem immensen Arbeitsprogramm nur andeuten konnte, deren Durchführung er aber einer kommenden Generation überlassen mußte.

\section{Zusammenfassung}

Zusammenfassend darf man sagen, daß es - obschon in früheren Jahrhunderten vereinzelte Ansätze einer von den Behörden organisierten gewerblichen Hygiene vorhanden waren - der verantwortungsbewußte Glarner Arzt gewesen ist, der angesichts der Nöte der arbeitenden Bevölkerung in der Schweiz zu einer Reform ansetzte. Schulers Wirken im letzten Drittel des 19. Jahrhunderts bildete den Ausgangspunkt für die Entwicklung einer schweizerischen Arbeitshygiene. Auf dem Gebiete der Gesetzgebung ging die Schweiz dank seiner Energie mit dem Fabrikgesetz von 1877 allen anderen Industrieländern voran und legte den Grundstein zum Ausbau der 
eidgenössischen Arbeiterschutzgesetzgebung sowie zur Verwirklichung des Kranken- und Unfallversicherungsgesetzes. Die Bedeutung der Arbeitsmedizin, Arbeitsphysiologie, Arbeitspsychologie und der beruflichen Prophylaxe der Berufskrankheiten hatte er trotz vielseitiger Anzweiflung richtig erkannt und wurde somit zum Begründer der modernen Arbeitshygiene in unserem Lande. In der Person Schulers ist der Urheber der Sozialmedizin zu finden, die heute dank der Konjunktur und der endlichen Aufgabe der von der Ärzteschaft geübten Zurückhaltung - teilweise wenigstens - einen beachtlich hohen Stand erreicht hat.

Wenn man heute im ganzen Lande die lichten, weiten, in frohen Farben gehaltenen, meistens in Grünanlagen mit Blumenbeeten gelegenen Fabriken betrachtet, denen abends eine saubere, geordnete und gut genährte Belegschaft entströmt, so kann man erfassen, welche gewaltige Entwicklung der soziale Gedanke und das Zusammengehörigkeitsgefühl in unserem Volke gefunden haben. Es ist das stolze Erbe eines großen Arztes und eines sozial denkenden Mannes, der im Glarnerlande seine Heimat hatte.

\section{Bibliographie}

Die Glarner Baumwollindustrie und ihr Einfluß auf die Gesundheit der Arbeiter, Dtsch. Vjschr. Gesundheitspflege 4 (1872).

Das eidgenössische Fabrikgesetz, von einem Glarner, Winterthurer Landbote 1877.

Kurze Geschichte des Landes Glarus, Glarus 1880.

Über die Ernährung der Fabrikbevölkerung und ihre Mängel, Schweiz. Z.f. Gemeinnützigkeit 21 (1882).

Die Ernährungsweise der arbeitenden Klassen in der Schweiz. (Mit besonderer Berücksichtigung der Alkoholfrage), Schweiz. Bundesblatt 1884.

Bericht über Gruppe 31, Hygiene, Balneologie und Rettungswesen, Schweiz. Landesausstellung Zürich 1883, Zürich 1884.

Die Leguminosen als Volksnahrung, Zürich 1885.

Untersuchungen über die Gesundheitsverhältnisse der Fabrikbevölkerung in der Schweiz (gemeinsam mit Albr. Burckhardt), Aarau 1889.

Die obligatorische Krankenversicherung in der Schweiz, Zürich 1891.

Das Chromblei in der Industrie, Corr. Bl. Schweiz. Ärzte 22 (1892).

Die Entwicklung der Arbeiterschutzgesetzgebung in der Schweiz, Brauns Arch. Soz. Gesetzgebung, Statistik 6 (1893).

Schutzvorrichtungen und Gewerbehygiene in ihren Beziehungen zur Unfall- und Krankheitsfrequenz, Z. Schweiz. Statistik 1894.

Die Fabrikwohnhäuser in der Schweiz, Z. Schweiz. Statistik 1896.

Die kantonalen Arbeiterinnenschutzgesetze in der Schweiz, ihr Vollzug und ihre Erfolge, Z. Sozialwiss. 2 (1899).

Das Verbot der Phosphorzündhölzchen, Berliner Tagblatt, 10. November 1900. 
Die Revision des schweiz. Fabrikgesetzes, o. O. 1902.

Über den Einfluß der Fabrikarbeit auf die geistige Entwicklung der Arbeiterschaft, $Z$.

Sozialwiss. 6 (1903).

Ausgewählte Schriften, Karlsruhe 1905.

\section{Biographische Literatur}

Dr. Fridolin Schuler, Erinnerungen eines Siebenzigjährigen, Frauenfeld 1903 (Porträt, Verzeichnis der Veröffentlichungen).

(Johannes) Seitz, Corr. Bl. Schweizer Ärzte 33 (1903) 474-9 (die Herausgabe der Autobiographie wurde wohl von SEITZ veranstaltet, der sich damit um die Schweizer Medizingeschichte verdient gemacht hat).

H. Wegmann, Die 50jährige Geschichte der Fabrikinspektion in der Schweiz, Arch. Gewerbepath. 5 (1934).

Erich AUer, Entwicklung und Stand der sozialmedizinischen Reform in der schweizerischen Industrie, Basler Veröffentlichungen zur Geschichte der Medizin und der Naturwissenschaften, Fasc. V., Basel 1955 (mit Bibliographie).

\section{Mitteilung}

$$
\text { I.P. V.Troxler (1780-1866) }
$$

Seit längerer Zeit wird eine Edition der Werke und Briefe des schweizerischen Arztes, Philosophen und politischen Publizisten Ignaz Paul Vital Troxler vorbereitet. Der Schweizerische Nationalfonds zur Förderung der wissenschaftlichen Forschung unterstützt die vorbereitenden Arbeiten mit namhaften Beiträgen. Das Kuratorium, das die Arbeiten zu überwachen und zu fördern berufen ist, bittet die Organe der schweizerischen und ausländischen öffentlichen und privaten Archive und Bibliotheken um Unterstützung des Herausgebers, Dr.theol. et phil. Emil Spiess, Hauterive (Post Posieux, Canton de Fribourg, Schweiz). Insbesondere bittet es alle diejenigen, die Troxlen-Dokumente besitzen oder von solchen Kenntnis haben, dem Herausgeber oder einem der Unterzeichneten davon Mitteilung zu machen.

Für das Kuratorium Troxler:

Prof.Dr. Georges Bonnard, Präsident der SGG Prof. Dr. Hans Barth, Universität Zürich Prof. Dr. Oscar Vasella, Universität Fribourg 